\title{
Regulierungen wirken sich auf die medizinische Versorgung aus
}

\author{
Patrick Müller \\ Leiter Abteilung Ambulante Versorgung und Tarife der FMH
}

\author{
Die steigenden Gesundheitskosten haben in den letzten Jahren zu zahlreichen \\ Regulierungen seitens der Politik geführt. Diese bleiben nicht ohne Folge für die \\ medizinische Versorgung. Eine repräsentative Studie zeigt erstmals die Ursachen \\ sowie die Auswirkungen auf die niedergelassene Ärzteschaft in der Schweiz auf. Sie \\ kann nachweisen, dass es Anzeichen einer fortschreitenden Kommerzialisierung \\ der Medizin gibt.
}

Die stetige Zunahme der Gesundheitskosten in der Schweiz und deren steigender Anteil am Bruttoinlandprodukt (BIP) in den letzten zwanzig Jahren haben zahlreiche politische Eingriffe und Regulierungen nach sich gezogen und den politischen Fokus zunehmend auf das Gesundheitswesen gelenkt [1]. Studien belegen, dass die zunehmende Steuerung und der Druck auf die Gesundheitskosten Auswirkungen auf die Erbringung von medizinischen Leistungen haben

Die zunehmende Steuerung und der Druck auf die Gesundheitskosten können Auswirkungen auf die Erbringung von medizinischen Leistungen haben.

können, indem betriebswirtschaftliche Faktoren medizinische Entscheidungen und Angebote beeinflussen können [2]. In diesem Zusammenhang werden in der Literatur die Begriffe Ökonomisierung und Kommerzialisierung der Medizin genannt. Dabei kann Ökonomisierung verstanden werden als ein Vordringen von wirtschaftlichen Prinzipien wie Effizienz, optimaler Ressourceneinsatz und Kostenbewusstsein in Bereiche, in denen vormals andere, eigene Prinzipien relevant waren [3]. Der Begriff Kommerzialisierung wird als die am weitest ausgebildete Form der Ökonomisierung angesehen und strebt dabei nach einer Gewinnmaximierung und einem selektiven Angebot in einem wettbewerbsorientierten Markt [4, 5]. Bezogen auf die Medizin kann dies zum Beispiel dazu führen, dass die verfügbare Zeit pro Patient kürzer wird, dass mehr Patienten pro Arzt und pro Tag behandelt werden [4], dass das Leistungsangebot nach der Abgeltung ausgerichtet wird [6] und dass der Fokus auf die Kosten der Leistungserbringung gelegt wird [7]. Weitere Folgen können sein, dass mehr Selbstzahlerleistungen angeboten werden [6] oder eine Patientenselektion betrieben wird, indem komplexe Patienten vermehrt an andere Leistungserbringer überwiesen werden [4]. Weiter wird in der Literatur beschrieben, dass diese Entwicklungen mit der zunehmenden Bürokratisierung und Überadministrierung der ärztlichen Tätigkeit einhergehen, da nur das bezahlt wird, was dokumentiert und nachgewiesen werden kann. Dazu kommen die vielen Rückfragen seitens der Kostenträger [8]. Dies geht meistens mit einer höheren Arbeitsbelastung, mehr Arbeitsstunden und damit verbunden auch mit einer Abnahme der Arbeitszufriedenheit einher [2]. In der einschlägigen Literatur geht man davon aus, dass die Entstehung der Kommerzialisierung der Medizin nicht aus dem Eigenantrieb der Ärztinnen und Ärzte erfolgt ist, sondern durch gesundheitspolitische Fehlsteuerungen und Fehlanreize entstanden ist, weil der Wettbewerb im Gesundheitswesen vielfach über Tarife oder Kosten stattfindet [7].

Der Autor hat im Rahmen eines postgradualen Studiengangs zum Executive Master of Business Administration (Executive MBA) in einer Masterarbeit zum Thema «Kommerzialisierung der Medizin und ihre Auswirkungen auf die niedergelassenen Ärztinnen und Ärzte in der Schweiz» untersucht, welchen Einfluss die Kommerzialisierung resp. Ökonomisierung der Medizin auf die Arbeitszufriedenheit, das betriebswirtschaftliche Wissen der Ärztinnen und Ärzte und auf die Praxisorganisation der selbständig erwerbenden niedergelassenen Ärztinnen und Ärzte in der Schweiz hat. Dabei wurde auch untersucht, ob ökonomische Ziele und Vorgaben einen Einfluss auf die Art der Patientenbehandlung, das Leistungsangebot, die 
Arbeitszeiten, die Zufriedenheit und die Praxisstruktur der niedergelassenen Ärztinnen und Ärzte haben und ob zwischen den Facharztdisziplinen Unterschiede betreffend diese Auswirkungen erkennbar sind. Dazu wurde ein fundiertes Literaturstudium als Basis durchgeführt. Anschliessend erfolgte eine qualitative Erhebung mittels Leitfadeninterviews mit ausgewählten niedergelassenen Ärztinnen und Ärzten mit unterschiedlichen Facharzttiteln, welche auch als wichtige Grundlage für die nachfolgende quantitative Erhebung diente. In der repräsentativen quantitativen Erhebung mittels einer Onlineumfrage haben vom 11. April 2019 bis 16. Mai 2019 insgesamt 3900 niedergelassene Ärztinnen und Ärzte mit eigener Praxis oder Teilhaber aus der ganzen Schweiz teilgenommen, davon konnten 3618 qualifizierte und valide Datensätze für die statistischen Auswertungen verwendet werden. Um die in der Forschungsfrage angesprochene mög-

\section{An der Onlineumfrage haben 3900 niedergelas-} sene Ärztinnen und Ärzte mit eigener Praxis oder Teilhaber aus der ganzen Schweiz teilgenommen.

liche Differenz zwischen den Facharzttiteln zu untersuchen, wurden diese in drei Facharztgruppen zusammengefasst, welche auf einer vom BAG definierten Gruppierung beruhen [10]. Die erste Gruppe, «Grundversorgung», enthält die Weiterbildungstitel «Allge-

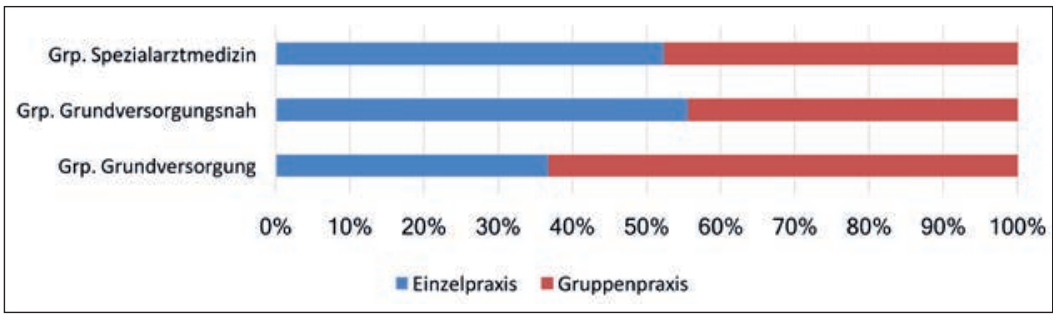

Vergleich der Verteilung der Praxisform zwischen den Facharztgruppen, $\mathrm{N}=3618$, eigene Darstellung.

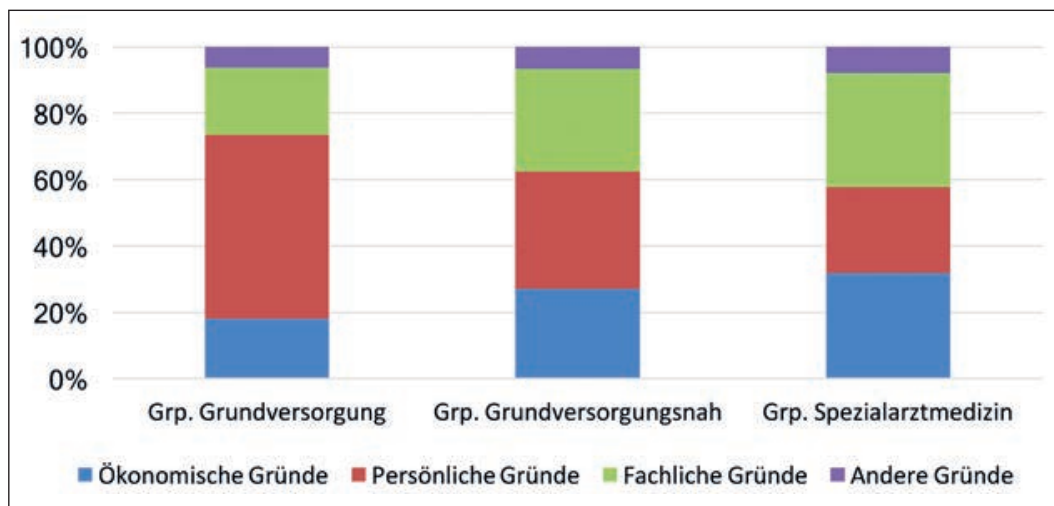

Primäre Hauptgründe für die Wahl als Gruppenpraxis, N = 1970, eigene Darstellung. meine Innere Medizin», «Kinder- und Jugendmedizin» und «praktischer Arzt», die zweite Gruppe, «Grundversorgungsnah», die Facharzttitel «Kardiologie» und "Gynäkologie/Geburtshilfe» und die dritte Gruppe, "Spezialarztmedizin», sämtliche restlichen Facharzttitel. Die Auswertungen erfolgten daher pro Fragestellung primär über diese drei Facharztgruppen.

\section{Gruppenpraxis immer beliebter}

Aus der Ärztestatistik der FMH 2018 [11] geht hervor, dass die Form der Gruppenpraxis immer beliebter wird. Die Auswertungen der Umfrage dieser Masterarbeit bestätigen diesen Trend. Heute sind bereits mehr als die Hälfte aller niedergelassenen Ärztinnen und Ärzte in der Schweiz in einer Gruppenpraxis tätig (54,45 Prozent). Dabei zeigen die Auswertungen, dass Grundversorger deutlich häufiger in einer Gruppenpraxis tätig sind. Ebenso sind Ärztinnen und Ärzte aus der Deutschschweiz deutlich häufiger (57,23 Prozent) in einer Gruppenpraxis tätig als ihre Kollegen in der Romandie (48,78 Prozent) oder im Tessin (38,46 Prozent). Die Umfrage untersuchte auch, welcher Hauptgrund ausschlaggebend war, für die Wahl der Tätigkeit in einer Gruppenpraxis. Aus den Resultaten geht hervor, dass persönliche Gründe den Hauptausschlag (42,47 Prozent) geben. Unter persönlichen Gründen kann beispielsweise eine verbesserte Work-LifeBalance, die Möglichkeit der Teilzeitarbeit oder eine bessere Stellvertretungsmöglichkeit verstanden werden. Dabei sind persönliche Gründe bei Ärztinnen (51,39 Prozent) häufiger ausschlaggebend als bei Ärzten (27,64 Prozent). Bei der Gruppe «Spezialarztmedizin» stehen primär fachliche Gründe, wie beispielsweise die Möglichkeit, ein breiteres medizinisches Angebot, der fachliche Austausch oder eine Teamarbeit ähnlich wie im Spital, im Vordergrund für die Wahl der Praxisstruktur.

\section{Erweiterung des Medizinstudiums um betriebswirtschaftliche Module}

Vier von fünf Ärztinnen und Ärzten sind der Meinung, dass das Medizinstudium oder die anschliessende Weiterbildung am Spital oder in der Praxis betriebswirtschaftliche Aspekte beinhalten sollte. Sämtliche befragten Ärztinnen und Ärzte in den qualitativen Interviews haben die Tatsache, dass dies heute nicht der Fall ist, als eindeutigen Missstand bezeichnet und waren der Auffassung, dass die jungen Ärztinnen und Ärzte damit zu wenig auf eine selbständige Erwerbstätigkeit vorbereitet werden. Die Vermutung, dass ein solches betriebswirtschaftliches Basiswissen wichtig 


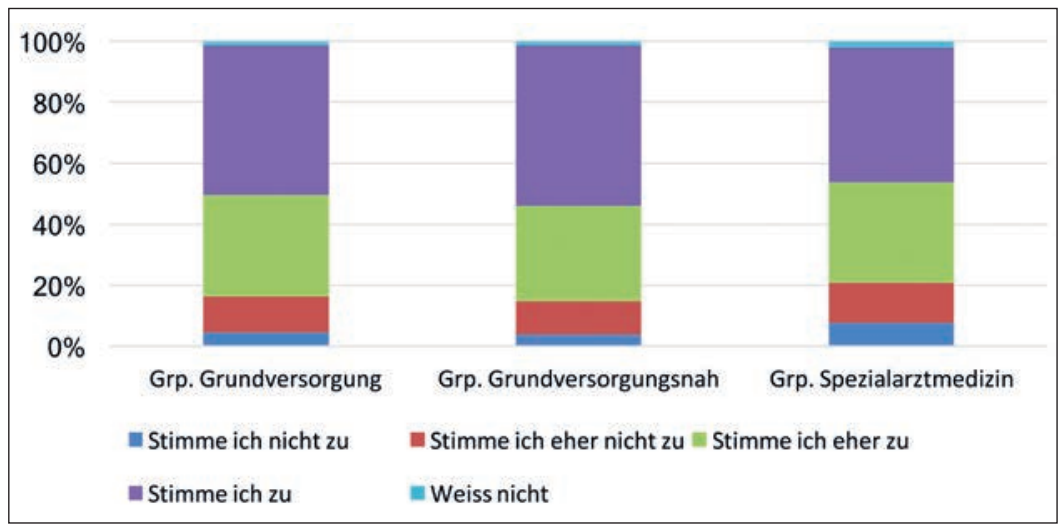

Antworten auf die Frage, ob das Medizinstudium oder die anschliessende Weiterbildung betriebswirtschaftliche Aspekte ausbilden soll, $\mathrm{N}=3618$, eigene Darstellung.

für das erfolgreiche Führen einer Arztpraxis ist, bestätigten wiederum vier von fünf Ärzten in der Umfrage. Die Auswertung ergab, dass nur eine von fünf Ärztinnen bzw. einer von fünf Ärzten eine betriebswirtschaftliche Weiterbildung im Anschluss an das absolvierte Medizinstudium absolviert hat. Dieser niedrige Anteil und der Umstand, dass vier von fünf Ärztinnen und Ärzten betriebswirtschaftliche Kenntnisse als wichtig erachten, bestätigen die Wichtigkeit der Vermittlung von betriebswirtschaftlichen Kenntnissen oder Managementfähigkeiten im Medizinstudium oder in der anschliessenden Weiterbildungszeit im Spital oder in der Praxis.

\section{Tarife führen zu Anpassungen am Leistungsangebot}

Die befragten Ärztinnen und Ärzte gaben in der Onlineumfrage an, dass im Vergleich zum Jahr 2014 die Wartezeiten für Patienten in Bezug auf einen Termin bei allen Facharztgruppen zugenommen haben. Weniger Zusatztermine wurden am Abend oder am Wochenende angeboten, und gleichzeitig ist die Anzahl Arbeitsstunden deutlich gestiegen. Die Auswertung lässt den Schluss zu, dass aufgrund der gestiegenen Arbeitsstunden die Bereitschaft zum Anbieten von zusätzlichen Abendterminen oder am Wochenende gesunken ist oder die frei gewordenen Zeitfenster bereits durch administrative Tätigkeiten besetzt sind. Die Auswertungen zeigen weiter, dass gegenüber dem Jahr 2014 Patientinnen und Patienten eher an weitere Spezialärztinnen und Spezialärzte oder an Spitäler überwiesen wurden. Im Detail zeigen die Auswertungen, dass sich die Gruppe "Spezialarztmedizin" mit 22,79 Prozent im Vergleich zur Gruppe «Grundversorgung» mit 16,47 Prozent häufiger auf ein gewisses Leistungsangebot spezialisiert und insbesondere bei der
Erweiterung um Nichtpflichtleistungen ebenfalls mit 22,79 Prozent deutlich mehr angepasst hat als die Gruppe "Grundversorgung» (16,47 Prozent) und die Gruppe «Grundversorgungsnah» (20,58 Prozent). Jede dritte Ärztin bzw. jeder dritte Arzt hat unrentable Leistungen aus seinem Portfolio gestrichen. Zudem ist ersichtlich, dass nur eine resp. einer von dreissig Ärztinnen und Ärzten Behandlungen, Untersuchungen oder Eingriffe aufgrund besserer Abgeltung den möglichen konservativen, alternativen Möglichkeiten vorziehen. Nur jede zehnte Ärztin oder jeder zehnte Arzt plant die Behandlungen, Untersuchungen, Eingriffe oder Beratungen der Patientinnen und Patienten so, dass diese tarifarisch am optimalsten abgebildet sind. Grund für die Anpassung des persönlichen Leistungsangebots war bei rund 32 Prozent der Ärztinnen und Ärzte die Deckung der steigenden Fixkosten (Personal, Infrastruktur, Medizintechnik, Material), mit dem Ziel, die Reduktion des persönlichen Einkommens zu minimieren. 26 Prozent gaben an, dass sie dies zur Kompensation der sinkenden Tarife tun, mit dem Ziel, das persönliche Einkommen auf einem ähnlichen Niveau halten zu können.

Jede siebte Ärztin oder jeder siebte Arzt gibt an, dass er in den letzten zwei Jahren zwecks Kostenoptimierung nichtärztliches Personal abbauen musste. Gerade bei niedergelassenen Ärztinnen und Ärzten stellen die nichtärztlichen Personalkosten den grössten Anteil der Fixkosten dar [12]. Die Reduktion von nichtärztlichem Personal kann dann ebenfalls Konsequenzen auf die eigene Arbeitsbelastung oder auf die Anpassung der Betriebszeiten haben, was sich wiederum in einer tieferen Arbeitszufriedenheit niederschlagen kann.

\section{Zunehmende Forderungen der Patien- tinnen und Patienten}

Mehrere Ärztinnen und Ärzte gaben in der qualitativen Erhebung an, dass sich das Arzt-Patienten-Verhältnis aus ihrer Sicht in den letzten Jahren verändert hat. Einerseits seien die Patienten immer besser über Behandlungsmethoden informiert, andererseits kämen sie bereits mit bestimmten Vorstellungen zum Arzt. Einige Ärztinnen und Ärzte berichten davon, dass Patienten an einen Spezialisten überwiesen werden wollen, den behandelnden Arzt auffordern, weitere medizinisch nicht notwendige Abklärungen oder Untersuchungen durchzuführen, oder den Arzt unter Druck setzen, eine Nichtpflichtleistung als Pflichtleistung abzurechnen. Die Auswertungen aus der Umfrage zeigen, dass die Gruppe "Grundversorgung» deutlich häufiger damit konfrontiert ist (20,81 Prozent «immer» oder «häufig») 


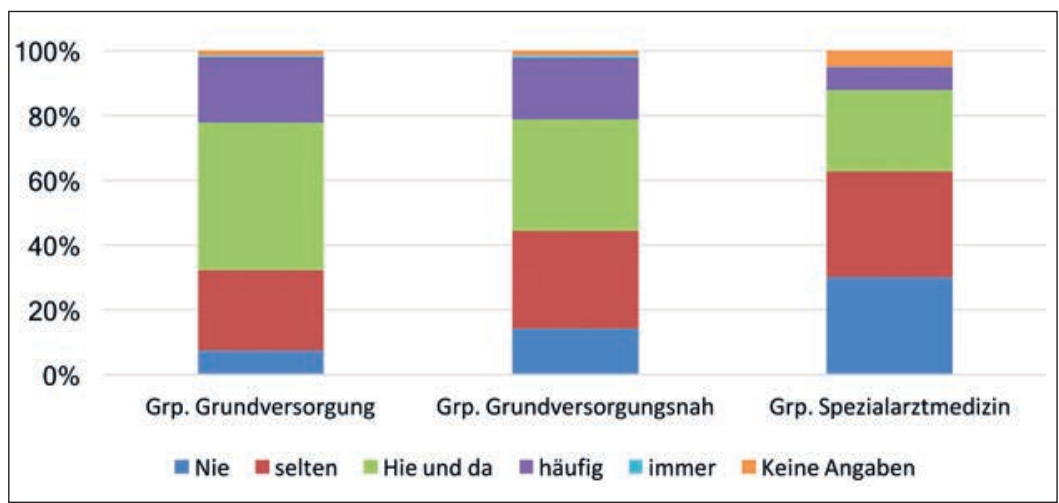

Antwort auf die Frage, wie häufig Patientinnen und Patienten Behandlungen, Untersuchungen, Eingriffe oder Beratungen, die medizinisch nicht notwendig wären, vom Arzt verlangen oder ihn auffordern, Nichtpflichtleistungen als Pflichtleistungen abzurechnen, $\mathrm{N}=3618$

\section{FMH}

Abteilung Ambulante Versorgung und Tarife

Baslerstrasse 47

CH-4600 Olten

Tel. 0313591230

tarife.ambulant[at]fmh.ch als ihre Kollegen der Gruppe «Spezialarztmedizin» (7,08 Prozent «immer» oder «häufig»).

\section{Attraktivität der niedergelassenen ärztlichen Tätigkeit sinkt}

Die interviewten Ärztinnen und Ärzte in der qualitativen Erhebung gaben an, dass aus ihrer Sicht die Arbeitsattraktivität für die niedergelassene Ärzteschaft seit ihrem Einstieg in eine eigene Praxis kontinuierlich abgenommen hat. Sie nannten dabei die zunehmenden Regulierungen und Vorgaben, den erhöhten Kostendruck und die verlängerten Arbeitszeiten als Gründe für die gesunkene Attraktivität. Weiter wurde angegeben, dass die Attraktivität als selbständig tätiger Arzt in der Praxis auch abgenommen hat, weil die in den letzten Jahren verbesserte Lohnsituation in den Spitälern sowie die gesetzliche Einführung der Höchstarbeitszeit die Arbeitsbedingungen in den Spitälern deutlich verbesserten. Zudem merkte ein Arzt an, dass das Einkommen bei gleichem oder höherem Arbeitseinsatz rückläufig ist. Die Auswertungen der Umfrage zeigen bei der Frage nach der Arbeitszufriedenheit einen Mittelwert von knapp 60 (Median = 70, $S D=28,64$ ), wobei 100 «ausserordentlich zufrieden" und 0 «ausserordentlich unzufrieden" bedeutet. Dabei ist die Facharztgruppe «Grundversorgung» zufriedener als die anderen beiden Facharztgruppen. Als Gründe für die zunehmende administrative Belastung wurden insbesondere die grosse Anzahl an Rückfragen, Berichtsanforderungen der Versicherungen oder die zunehmende Dokumentationsarbeit (beispielsweise elektronisches Patientendossier EPD oder Registerpflege) angegeben. Andere Studien belegen, dass die zunehmende administrative Belastung die Unzufriedenheit der Ärztinnen und Ärzte fördert [13]. Vier von fünf Ärztinnen und Ärzten möchten nicht in ein Angestelltenverhältnis wechseln, und ebenso viele würden den eingeschlagenen Weg in die Selbständigkeit wieder wählen.

\section{Fazit und Empfehlungen}

Die Arbeit kann nachweisen, dass die ambulante medizinische Versorgung durch niedergelassene Ärztinnen und Ärzte in der Schweiz Anzeichen einer fortschreitenden Kommerzialisierung aufweist, beispielsweise in Form einer zunehmenden Spezialisierung, erhöhter administrativer Belastung oder abnehmender Arbeitszufriedenheit. Dies kann als Folge der zunehmenden politischen Regulierungen und Eingriffe auf Vergütungs- oder Kostenebene im Gesundheitswesen gesehen werden. Die Arbeit formuliert fünf Empfehlungen, welche aus den Erkenntnissen abgeleitet werden können. Die wichtigsten Empfehlungen sind dabei die Aufnahme von betriebswirtschaftlichen Modulen ins Curriculum des Medizinstudiums und die Stärkung der Attraktivität der niedergelassenen ärztlichen Tätigkeit. Weiter sollten die Auswirkungen der Veränderung der Praxisstrukturen (Zunahme Gruppenpraxen) untersucht und Massnahmen zur Verminderung einer Überversorgung entwickelt werden.

\section{Hinweis}

Die vollständige Arbeit kann beim Autor angefordert werden.

\section{Literatur}

1 Bundesamt für Statistik BFS. Kosten und Finanzierung des Gesundheitswesens seit 1960. Neuenburg; 2018.

2 Braun B, et al. Pauschalpatienten, Kurzlieger und Draufzahler Auswirkungen der DRGs auf Versorgungsqualität und Arbeitsbedingungen im Krankenhaus (1. Ausg.). Bern: Hans Huber Verlag; 2010.

3 Krönig F. Die Ökonomisierung der Gesellschaft. Bielefeld: transcript Verlag; 2007.

4 SAMW. Medizin und Ökonomie-wie weiter? Basel; 2014

5 Schimank U, Volkmann U. Ökonomisierung der Gesellschaft. In: Schimank U, Volkmann U, Maurer A (Hrsg.). Handbuch der Wirtschaftssoziologie (1. Ausg.). Wiesbaden; 2008

6 Karsch F. Medizin zwischen Markt und Moral. Bielefeld: transcript Verlag; 2015.

7 Porter M, Teisberg Olmstedt E. Redefining Health Care. Massachusetts: Harvard Business School; 2006.

8 Rakowitz N. Europäisches Manifest gegen die Kommerzialisierung des Gesundheitswesens Health professionals erheben ihre Stimme. Maintal: Verein demokratischer Ärztinnen und Ärzte; 2012.

9 Naegler H, Wehkamp KH. Medizin zwischen Patientenwohl und Ökonomisierung. Berlin: Medizinisch Wissenschaftliche Verlagsgesellschaft mbH \& Co. KG; 2018.

10 Bundesamt für Gesundheit BAG. Zur aktuellen Situation der ärztlichen Grundversorgung. Bern; 2010.

11 FMH. FMH-Ärztestatistik. Bern; 2018.

12 Kraft E. Der Bund und die FMH kommen auf das gleiche Resultat. Schweiz Ärzteztg. 2018;99(43):1480-1.

13 Willner T. Vom Halbgott in Weiss zum Unternehmer: Chefärzte und die Ökonomisierung des Gesundheitswesens. St. Gallen: Universität St.Gallen, Hochschule für Wirtschafts-, Rechts- und Sozialwissenschaften sowie Internationale Beziehungen (HSG); 2012. 